\title{
Article
}

\section{My heart is racing! Psychophysiological dynamics of skilled racecar drivers}

Filho, Edson, Di Fronso, Selenia, Mazzoni, Caterina, Robazza, Claudio, Bortoli, Laura and Bertollo, Maurizio

Available at http://clok.uclan.ac.uk/14050/

Filho, Edson ORCID: 0000-0002-8548-4651, Di Fronso, Selenia, Mazzoni, Caterina, Robazza, Claudio, Bortoli, Laura and Bertollo, Maurizio (2015) My heart is racing! Psychophysiological dynamics of skilled racecar drivers. Journal of Sports Sciences, 33 (9). pp. 945-959. ISSN 0264-0414

It is advisable to refer to the publisher's version if you intend to cite from the work. http://dx.doi.org/10.1080/02640414.2014.977940

For more information about UCLan's research in this area go to http://www.uclan.ac.uk/researchgroups/ and search for <name of research Group>.

For information about Research generally at UCLan please go to http://www.uclan.ac.uk/research/

All outputs in CLoK are protected by Intellectual Property Rights law, including Copyright law. Copyright, IPR and Moral Rights for the works on this site are retained by the individual authors and/or other copyright owners. Terms and conditions for use of this material are defined in the policies page. 


\section{1}

\section{4}

5

\title{
My heart is racing! Psychophysiological dynamics of skilled racecar
}

\author{
drivers
}

Edson Filho ${ }^{\mathrm{a}}$, Selenia Di Fronso ${ }^{\mathrm{a}}$, Caterina Mazzoni ${ }^{\mathrm{a}}$, Claudio Robazza ${ }^{\mathrm{a}}$ Laura Bortoli $^{\mathrm{a}}$ \& Maurizio Bertollo ${ }^{\mathrm{a}}$

${ }^{a}$ BIND - Behavioral Imaging and Neural Dynamics Center, Department of Medicine and Aging Sciences, University of Chieti, Chieti, Italy.

Corresponding Author:

Edson Filho

University of Central Lancashire

School of Psychology

Darwin Building, DB114

Preston

Lancashire, United Kingdom

PR1 2HE

efilho@uclan.ac.uk

Running title: Psychophysiological dynamics of skilled drivers

Keywords: MAP model, psychophysiology, motorsport, peak performance

Number of figures: 4 
Abstract

2 Our purpose was to test the multi-action plan (MAP) model assumptions in which athletes'

3 psychophysiological patterns differ among optimal and suboptimal performance experiences.

4 Nine professional drivers competing in premier race categories (e.g., Formula 3, Porsche GT3

5 Cup Challenge) completed the study. Data collection involved monitoring the drivers' perceived

6 hedonic tone, accuracy on core components of action, posture, skin temperature, respiration rate,

7 and heart rate responses during a 40-lap simulated race. Time marks, gathered at three

8 standardized sectors, served as the performance variable. The A1GP racing simulator (Allinsport,

9 Modena) established a realistic race platform. Specifically, the Barcelona track was chosen due

10 to its inherently difficult nature characterized by intermittent deceleration points. Idiosyncratic

11 analyses showed large individual differences in the drivers' psychophysiological profile, as well

12 as distinct patterns in regards to optimal and suboptimal performance experiences. Limitations

13 and future research avenues are discussed. Action (e.g., attentional control) and emotion (e.g.,

14 biofeedback training) centered applied sport psychology implications are advanced.

15

16 Key words: MAP model, psychophysiology, motorsport, peak performance 


\section{My Heart is Racing! Psychophysiological Dynamics of Skilled Racecar Drivers}

Research on expertise in sport has been directed at identifying psychophysiological mechanisms underlying consistently high performance levels (Ericsson, 2006; Hanin \& Hanina, 2009). Although nomothetic frameworks are essential to the development of general guidelines on expertise, idiosyncratic models are paramount in applied sport psychology (Bertollo et al., 2012; Hanin \& Hanina, 2009; Robazza, 2006). To this extent, various idiosyncratic frameworks have been adopted by practitioners working with athletes to enhance performance. Recently, Bortoli, Bertollo, Hanin, and Robazza (2012) proposed the multi-action plan (MAP) model which, like other models in applied sport psychology (e.g., mindfulness-acceptance-commitment approach, individual zones of optimal functioning, and optimal experience), reflects an idiosyncratic and multidimensional approach to performance enhancement in sport (Gardner \& Moore, 2004; Hanin, 1997; Kimiecik \& Jackson, 2002). The unique contribution of the MAP model pertains to its parsimonious $2 \times 2$ conceptualization on how performance levels interact with attentional control levels. Parsimonious models are important because under competitive pressure athletes are more likely to attend to simple and clear instructions rather than complex and difficult information (Tenenbaum, Basevitch, Gershgoren, \& Filho, 2013).

The MAP model's $2 \times 2$ organization (see Figure 1 ) has been conceptualized to offer clear “multi-performance enhancement plans” according to four performance types. Type 1 performance is characterized by automatic attentional control and optimal performance. This state involves optimal, flow-like performance experiences and low overt conscious control on the action. Type 2 performance is typified by attentional focus directed at athletes' core components of action and functional performance. This performance is attained through consciously focused attention on critical components of the task, such as pedaling rate in cycling or aiming in 
shooting sports. Type 3 performance is characterized by serial processing/over-controlled

2 attention, and dysfunctional performance. The excessive reinvestment of attention on the task in

3 the attempt to control execution undermines automaticity and ultimately leads to poor

4 performance. Type 4 performance is typified by low-level or task-irrelevant attentional focus,

5 insufficient energies deployed to complete the task, and dysfunctional performance. Previous empirical research on the MAP model reinforces the notion that addressing the

7 "performance-attentional control linkage" may be instrumental in the development of multi-plans 8 for performance enhancement during competition. For instance, Comani et al. (2014b) observed

9 that action strategies directing athletes' attentional focus to previously identified core

10 components of action, such as cycling pace and pedaling rate, lead to performance improvement

11 in endurance cycling. In a study with skilled pistol and rifle shooters, Robazza, Bertollo, Hanin,

12 Filho, and Bortoli (2014) found different probability curves (see Kamata, Tenenbaum, \& Hanin, 13 2002) linked to the four different performance typologies proposed in the MAP model.

14 Moreover, Bertollo, Bortoli, Gramaccioni, Hanin, Comani, and Robazza (2013) observed that 15 skilled shooters' heart rate and skin conductance level were lower for Type 1 performance, when 16 compared to suboptimal performance types. Finally, in another psychophysiological study,

17 Comani et al. (2014a) observed that the neural correlates underlying the MAP model's $2 \times 2$

18 performance were different, with optimal performance states (Type 1 and Type 2) characterized

19 by the quiescence of the motor cortex in agreement with the neural efficiency hypothesis.

20 According to this hypothesis, skilled performance is characterized by fewer unnecessary

21 communications among brain cortices, resulting in less energy expenditure and interference in

22 motor responses (see Comani, 2014a; Del Percio et al., 2009). 
It is important to note that previous research on the MAP model has targeted objective

2 performance measures of skilled shooting and endurance cycling athletes (Bortoli et al., 2012;

3 Comani et al., 2014a, 2014b). To this extent, Hanin (2007) observed that objective performance measures allow for reliable estimates of one’s moment-to-moment performance fluctuations, as

5 related to a myriad of psychophysiological variables. Furthermore, skilled athletes show greater awareness of their idiosyncratic core components of action linked to peak performance in sports

7 (Ericsson, 2006; Hanin \& Hanina, 2009). Specific to objectively measured sports, skilled racecar drivers have shown greater awareness of strategic (e.g., route timing and journey) and tactical

9 (e.g., maneuvering, compensatory breaking) knowledge linked to safety and optimal

10 performance (Fuller, 2005). In the present study, we tested the MAP model assumptions among

11 highly skilled racecar drivers. We considered an objective performance measure and a

12 multimodal approach by targeting multiple psychophysiological variables. In this regard,

13 Bertollo et al. (2013) recently emphasized the importance of testing the MAP model assumptions

14 in sport modalities other than self-paced sports, such as dart throwing, rifle, and pistol shooting, 15 and especially in open and complex skill sports.

In addition to testing the MAP model assumptions in a new sport modality, the present

17 study addressed the need for further research in motorsports (Potkanowicz \& Mendel, 2013).

18 Compared to other traditional sports (e.g., cycling, track and field athletics, water sports), few

19 studies exist in racecar driving (Yamakoshi, Matsumura, Yamakoshi, Hirose, \& Rolfe, 2010).

Data collection during actual racing may interfere with one’s safety, thus imposing a challenge to

21 scholars interested in motorsports (Fuller, 2005). However, relatively recent advances in

22 bioengineering have allowed scholars to safely use portable electro-physiological sensors in the

23 real-time monitoring of racecar drivers (Katsis, Katertsidis, Ganiatsas, \& Fotiadis, 2008; 
Potkanowicz \& Mendel, 2013). In fact, recent studies on simulated racecar driving have focused on recording racecar drivers’ psychophysiological signals, including heart rate, respiration rate, and body temperature (Edmonds, Tenenbaum, Mann, Johnson, \& Kamata, 2008; Mullen, Jones, Faull, \& Kingston, 2012; Yamakoshi et al., 2010).

Previous psychophysiological studies on racecar drivers have centered on monitoring: (a) heart rate dynamics, (b) thermal stress, and (c) body posture intrinsically related to the cars' ever changing momentum resulting from acceleration and braking (Katsis et al., 2008). Specifically, heart rate has been shown to be positively related to various psychophysiological stressors common to motorsports, including exercise intensity, risk-taking behavior, arousal, and dehydration (Brearley \& Finn, 2007; Yamakoshi et al., 2010). Respiratory rate is another psychophysiological marker of arousal regulation, and as such breathing control exercises are among the most common forms of biofeedback training (Giggins, Persson, \& Caulfield, 2013). Thermal stress has also been studied in racecar driving (Walker, Dawson, \& Ackland, 2001). The numerous safety garments and layers of clothing worn by drivers, in addition to the heat generated by the car engine, creates a microenvironment that can reach $50^{\circ} \mathrm{C}$ and compromise drivers' thermoregulation capability (Katsis et al., 2008). Finally, drivers' body posture is influenced by the cars’ ever changing momentum resulting from acceleration and braking. Posture data can be either positive or negative and is usually measured on more than one axis, such as body flexion-extension and arms abduction/adduction in more than one axis (Potkanowicz \& Mendel, 2013). In the present study, we expanded upon prior research by simultaneously monitoring skilled drivers' heart rate response, body temperature, and posture movement. 
Although research has shown that psychophysiological monitoring is important to understand racecars’ performance, sport scientists also widely agree that it is essential to consider drivers’ perceived emotional states (Edmonds et al., 2008; Fuller, 2005). Specifically, there is a general agreement that perceived psychological states influence performance, which in turn affect individuals’ emotional states (for a review, see Tenenbaum et al., 2013). Relying only on objective data may misrepresent various situational factors outside one’s control, including bad weather, injury, mechanical problems, and outstanding performance by opponents. Furthermore, given that racecar drivers sit alone in the cockpit during races, Potkanowicz and Mendel (2013) noted that behavioral observations (from spectators, coaches, and scientists) are limited, and self-reports are paramount in assessing drivers’ inner thoughts. Holland, Geraghty, and Shah (2010) highlighted that perceived control predicts driving behavior among male and female drivers. Edmonds et al. (2008) found that perceived affective states (i.e., arousal and hedonic tone) were reliable predictors of optimal, moderate, and poor performance in a simulated car racing study. Fuller (2008) observed that perceived performance and subjective risk appraisal influence compensatory speed reductions, which in turn effects performance and safety in motorsports. In particular, Fuller noticed that drivers tend to drive faster when they perceive poor performance times. In the present study, we were interested in drivers' perceived accuracy on their core components of action and hedonic tone (i.e., pleasantness level ranging from very low to very high; see Russell, Weiss, \& Mendelsohn, 1989), given that these variables have been shown to be associated with performance and attentional focus in motorsports (Edmonds et al. 2008; Fuller, 2008; Mullen et al., 2012).

In summary, we subscribed to an idiosyncratic multi-modal approach by considering psychophysiological and perceived emotional states of skilled racecar drivers. Specifically, we 
conducted a multi-case study to test the MAP model's general assumption in which different psychophysiological characteristics underlie four different performance types (Figure 1). We considered an objective performance measure and a multimodal approach by targeting multiple psychophysiological variables. Consistent with previous research (Bertollo et al., 2013; Bortoli et al., 2012), we hypothesized that: (a) performance categories (Type 1, Type 2, Type 3, and Type 4) would differ according to athletes' self-reports (perceived performance, emotional states) and physiological recordings (heart rate response, respiratory rate, skin temperature, and posture); and (b) drivers’ psychophysiological responses would show idiosyncratic patterns, akin to previous idiographic research in sport psychology (Edmonds et al., 2008; Hanin 2007; Johnson, Edmonds, Moraes, Filho, \& Tenenbaum, 2007; Robazza, 2006).

\section{Method}

\section{Participants}

Ten male professional racecar drivers participated in the study. The participants ranged in age from 19 to 46 years $(M=29.1, S D=10.3)$, and had on average 9.9 years $(S D=4.75)$ of driving experience. We used a criterion sampling approach given that sample size is not crucial in idiosyncratic analyses (Hanin, 2007; Patton, 2002; Robazza, 2006). Specifically, we selected participants on the basis of their competitive experience and professional achievements in major racecar events. All participants were skilled drivers, consistent with the importance of studying information rich cases to advance knowledge on the underlying mechanisms of excellence across domains of human performance, such as attentional control through fixation and duration of visual scan strategies (see Tenenbaum et al., 2013; Vickers, 2006). Furthermore, skilled athletes, as opposed to novices, are more knowledgeable about the core components (i.e., chain of events, mediating factors) of skilled performance (Hanin \& Hanina, 2009). More specifically, the 
participants competed in premier categories including Formula 3, Formula 3 Open, Formula 3000, Lamborghini Super Series, Maserati World Series Championship, and Porsche GT3 Cup Challenge. Participants’ career highs included winning overall seasons and top-3 placements in the aforementioned events, as well as serving as Ferrari test-drivers on the Formula-1 team.

\section{Instrumentation}

Pre-task assessment: Verbal reports on core-components of action. Participants were asked to identify the core components of their driving action. Initially, the participants were encouraged to provide a rich and detailed description of the chain of actions linked to their best performances (i.e., fastest race laps). The participants were instructed to think aloud while describing in a step-by-step mode the cognitive, motor, emotional, and environmental aspects of their optimal driving behavior (Ericsson, 2006). Think aloud protocols have been successfully used to study expert performance across domains (Ericsson, 2006; Williams \& Ericsson, 2005). In particular, this methodology is based on the notion that experts are able to verbalize their cognitive processes linked to the successful completion of a given task. In the present study, we used the think aloud method as a means to identify the participants' core components of action. The verbal report sessions were conducted individually in an informal brainstorming tone in order to develop rapport. Upon finishing the description of the chain of actions linked to their unique performance dynamics, the participants were asked to select those elements (i.e., the core components) viewed as crucial in differentiating optimal from suboptimal performance. The specific probe was: "What are the actions or behaviors that, when executed in a less accurate manner, cause your performance time to drop from optimal to suboptimal levels?” We explained to the participants that core components of actions are idiosyncratic and not necessarily the technical or tactical aspects emphasized by the press, coaches, or their peers. We also explained 
that core components of action can be supervised with more or less conscious control depending on whether one is experiencing functional (Type 1 and Type 2) or dysfunctional performance (Type 3 and Type 4) (see Bertollo et al., 2013; Bortoli et al., 2012).

Driving task. Three driving tasks were established after two in-person peer debriefing meetings involving the authors of this study and a former professional racecar driver whom is currently a senior driving coach. The peer debriefing meetings, based on the notion of cognitive task analysis (Ericsson, 2006), were aimed at identifying a reliable and challenging task able to capture high-skilled performance in a realistic context. The authors and coach selected the Barcelona race track due to its inherently difficult nature with numerous turns and intermittent deceleration points. This race track is considered an important racecar circuit in Europe and wellknown by all participants. The Barcelona track has a total length of 4.65 kilometers and is used by various Formula-1 teams as a testing circuit because of its sectorial characteristics. Specifically, this race track has three distinct sectors of comparable length with five braking points of similar difficulty.

Noteworthy, the driving task was operationalized through the Allinsport 1 Grand Prix racing simulator (Allinsport, Italy). This virtual reality simulator is a replica of a real racecar with a seat, steering wheel, and pedals (brake and accelerator) built in real-world dimensions. The participants were able to regulate the height as well as the distance of their seats from the steering wheel. Of note, the Allinsport 1 Grand Prix does not have G-force simulating capability. However, the Allinsport 1 Grand Prix racing simulator creates a realistic race platform through the combination of multi-media technology (sound, video, and kinematic interfaces) projected during real-time on a rounded (180 degrees) high-definition screen monitor. The participants were asked to drive 40 uninterrupted laps (approximately one hour simulation). Performance 
data was recorded at the end of each of the three sectors (i.e., three times per lap) for the 40 laps, and thus a total of 120 data points were collected per participant. This is consistent with the central limits theory and previous idiosyncratic research in sport psychology (Filho, Moraes, \& Tenenbaum, 2008; Kamata et al., 2002), in which a minimum of 30 data points per performance category should be initially considered for analysis.

Performance measure. The total time to complete each sector was automatically recorded by the racing simulator and represented the performance measure in this study.

Attentional control. In addition to performance data, the drivers’ perceived attentional control on their core components of the action was collected to allow for the establishment of the four performance categories described in the MAP model. Throughout the driving task, the participants were asked to rate their control levels by using a modified 11-point Borg scale (see Borg, 2001) ranging from 0 (extremely inaccurate) to 11 (extremely accurate). More specifically, the verbal anchors of the scale, developed to avoid floor and ceiling effects, were 0 = nothing at all, $0.5=$ very, very little, $1=$ very little, $2=$ little, $3=$ moderately, $5=$ much, $7=$ very much, 10 = very, very much, 11 = maximal possible. No verbal anchors were used for 4, 6, 8 and 9. Of note, this scale has been successfully used in psychophysiological research in sport and exercise psychology (Bertollo et al., 2012, 2013).

Accuracy of core components of action. As presented herein, subjective accuracy reports are important in idiosyncratic research in applied psychology (Robazza, 2006; Tenenbaum et al., 2009). Accordingly, participants' perceived accuracy of the execution of their core components of action were also assessed on the modified 11-point Borg scale. Correlation coefficients between individual's perceived accuracy ratings and lap times ranged from .58 to .84 
1 (mean $r=.69$ ), thus indicating a moderate to high criterion related validity and suggesting that

2 perceived accuracy of core components was related to performance.

Hedonic tone. Driver's hedonic tone was also collected throughout the driving task using

4 the modified Borg scale ranging from -11 (extremely unpleasant) to 11 (extremely pleasant),

5 with a 0 score denoting neither a pleasant nor unpleasant state. Negative scores are attributed to

6 unpleasant states (Hanin, 2007; Robazza, 2006).

7 Psychophysiological data. Each driver’s heart rate, respiratory rate, posture data, and

8 skin temperature were monitored throughout the driving task A BioHarness belt device (Zephyr

9 Technology) wirelessly connected to a data acquisition device (Powerlab 16/30, ADInstruments,

10 Australia) and a laptop computer with Labchart 7.1 software (ADInstruments) captured the

11 participant's heart rate frequency (beats per minute), respiratory rate (number of breaths per

12 minute), temperature $\left({ }^{\circ} \mathrm{C}\right)$, and posture data on the longitudinal axis relative to the sternum (i.e.,

13 body flexion-extension with positive values representing movements frontwards and negative

14 values for movements backwards). Physiological data collection were synchronized with the

15 simulator via a Bayonet Neill-Concelman cable directly connected between the brake and the

16 Powerlab data acquisition system.

17 Procedure

One of the authors, with extensive professional networking in motorsports, contacted

19 potential participants through phone calls and email correspondence. During these initial correspondences, the participants were briefed on the overall purposes of the study and had their

21 concerns and questions fully addressed. Those drivers interested in taking part in the study were

22 invited to the driving center where the study took place over the course of two visits. During

23 their first visit to the driving center, the participants received additional information regarding 
the study's overarching purpose and signed a written informed consent approved by the author's university ethical review board. The participants were then individually asked about their core components of action related to their best performance experiences in racecar driving, with each session lasting approximately one hour. The verbal report sessions were conducted in a quiet and safe meeting room to ensure the comfort and privacy of the participants. Upon completion of each idiosyncratic verbal report, the drivers were given approximately five trial laps in the racing simulator. All drivers were accustomed to practicing in driving simulators. Thus, this driving routine was particularly conceived to allow the participants to become familiar with the study's data collection procedures.

During their second visit to the driving center, the participants were given three additional familiarization laps prior to the commencement of the actual driving simulation. After these three initial familiarization laps, the actual simulation started and the participants were asked to drive for a total of 40 uninterrupted laps, totaling approximately one hour of a driving simulation. They wore their personal racing suits but did not wear helmets in order to facilitate the collection of verbal reports during the simulated race. In particular, while driving, the participants were asked to verbally report (at the end of each sector) their perceived levels of control, hedonic tone, and accuracy on their core components of action. Gathering verbal reports during, rather than prior to or after, sporting events has been encouraged in the literature in order to reduce ecological validity threats (Filho et al., 2008; Hanin, 2007; Kamata et al., 2002). Moreover, collecting verbal reports during racecar simulation is ecologically valid as brief verbal communication among racecar drivers and their racing team is common practice during race events (see The perfect lap, documentary feature by Mclaren Mercedes, 2013). 
Furthermore, while performing the driving task, the participants had their heart rate, respiratory rate, skin temperature, and postural data monitored. Baseline data on all physiological measures were gathered for five minutes prior to the start of the driving task to ensure that the participants’ physiological responses were within normal ranges. A BioHarness lightweight strap, mounted directly below each driver's chest, was used to capture and transmit heart and respiratory rate to a wirelessly connected laptop. The BioHarness strap is portable technology similar to a standard polar heart rate monitor. This strap is able to capture heart rate, respiratory rate, temperature, and posture data. Noteworthy, three trained researchers collected the data, with two monitoring the BioHarness equipment and Powerlab software, and one monitoring the driving simulator and recording the drivers' verbalized self-report data (i.e., control, hedonic tone, and perceived accuracy on core components of action).

\section{Data Analysis}

The data analyses procedures consisted of three steps. First, the psychophysiological data were organized using the Labchart software version 7.1 and in respect to the three sectors of the race. Given that the unit of analysis was the race sector, the psychophysiological data were averaged accordingly. The performance data for each participant were standardized (Ztransformation) across the three sectors of the race track, thus resulting in 120 data points per participant. We also multiplied the performance data by -1 (given that a shorter time racing corresponds to a better performance) to allow for ease of interpretation.

The second step of the data analyses procedures consisted of coding the data in respect to the MAP model's $2 \times 2$ categorization (performance $\times$ control). The leading and last author coded the data, discussing any potential disagreement until reaching a consensus. Performance and control median scores were computed for each participant to conduct an idiosyncratic 
analysis. Of note, median values were used because mean values are more susceptible to the influence of outliers, particularly in idiographic analysis. Accordingly, values above the median for performance, and below the median for control, were coded "as optimal/automatic" experiences (i.e., Type 1 performance). Values higher than the median for both of these variables were coded as “optimal/controlled” (i.e., Type 2 performance). Values lower than the median for performance and higher than the median for control represented "suboptimal/over-controlled" experiences (i.e., Type 3 performance).

Finally, values lower than the median for both performance and control were coded as “suboptimal/under-controlled” experiences (i.e., Type 4 performance). This coding procedure is in agreement with general guidelines on idiosyncratic research on peak performance (Bortoli et al., 2012; Kamata et al., 2002). Furthermore, the coding procedure is intended to increase the likelihood of an approximately even frequency distribution across different functional (Type 1 and Type 2) and dysfunctional (Type 3 and Type 4) performance experiences. The final step consisted of comparing the drivers’ psychophysiological and self-report data in regards to the coded data based on the MAP model's $2 \times 2$ conceptualization. Specifically, one-way ANOVAs with the four MAP model's categories as the between factors was run for all psychophysiological and self-report variables considered in this study. pool because a malfunctioning wireless connection interfered with his data acquisition. Respiratory rate from two drivers (Driver 3 and Driver 7) showed unreliable patterns (i.e., unrealistic and chaotic ranges) and were thus excluded from further analysis. It is important to note that these intercurrences are proper to psychophysiological studies in motorsport due to 
drivers’ natural movements and various layers of clothing, among other factors (e.g., vibrations from the car simulator; see Yamakoshi et al., 2010). Altogether, we limited our analysis to the data collected and recorded reliably, and present our findings for each hypothesis.

Core components of action. Participants’ final selection of core components of action included “acceleration after the curve” $(n=3)$, "braking modulation” $(n=2)$, "braking point” $(n$ =2), “car speed”, “racing line”, and “turning in point”. These results suggest that there is some variability in what racecar drivers consider to be a key factor for optimal performance in motorsports. The ability to properly use the brakes as well as re-gaining speed "after the curve" were emphasized as important aspects of performance by various drivers.

Hypothesis 1. Descriptive and inferential statistics for each driver's perceived and psychophysiological responses are presented in Table 1 and 2, respectively. A series of one-way ANOVAs with Bonferroni post-hoc tests was used to identify potential differences among the MAP model's categories, and in respect to each driver's data. The magnitude of observed differences is reported (Cohen's $d$ ) for overall effects. Specific effects can be derived from the descriptive statistics presented in Tables 1 and 2. Overall, the data analyses revealed that all drivers exhibited different accuracy and hedonic tone responses for the MAP model's optimal (Type 1 and Type 2) suboptimal categories (Type 3 and Type 4). Differences between optimal/automatic (Type 1) and optimal/controlled (Type 2) categories were observed for driver 9 only (both accuracy and hedonic tone). Differences between suboptimal/over-controlled (Type 3) and suboptimal/under-controlled (Type 4) experiences were observed for drivers 5 (accuracy), 7 (hedonic tone), and 8 (accuracy).

Drivers’ psychophysiological responses also varied according to the MAP model's categorization (Table 2). At least one psychophysiological marker was found to differ across 
drivers, with some drivers exhibiting differences in all analyzed variables (i.e., Drivers 1, 2, 3, and 6). In fact, differences among optimal (Type 1 or Type 2) versus suboptimal performance experiences (Type 3 or Type 4) were observed for all drivers. Differences between optimal/automatic (Type 1) and optimal/controlled (Type 2) categories were observed for Drivers 3 and 6 for heart rate only. Differences between suboptimal/over-controlled (Type 3) and suboptimal/under-controlled (Type 4) experiences were observed for heart rate (Drivers 1, 5, and 6), respiratory rate (Driver 6), skin temperature (Drivers 1 and 4), and posture (Driver 1). Altogether, these results are congruent with the notion that the MAP model's categories are associated with different perceived and psychophysiological states. However, it is important to note that most of the observed differences were in the performance (optimal/suboptimal) factor.

Hypothesis 2. The drivers presented idiosyncratic intensities and ranges of perceived and psychophysiological responses related to the different MAP model's categories (see Figures 2, 3, and 4). As presented in Tables 1 and 2, the magnitude of these differences varied greatly from driver to driver (see Cohen's $d$ ranging from 0.09 to 3.87). Finally, although the number of performance experiences classified as functional (Type 1 and Type 2) and dysfunctional (Type 3 and Type 4) was approximately even for all drivers, they still differed in the frequency of experiencing Type 1, Type 2, Type 3, and Type 4 performances. Collectively, these results are in agreement with our second hypothesis, in which drivers’ self-reports and physiological recordings would show large inter-individual differences.

\section{Discussion}

Our purpose was to test the MAP model assumptions in which athletes’ psychophysiological patterns are thought to differ among optimal/automatic (Type 1), optimal/controlled (Type 2), suboptimal/over-controlled (Type 3), and suboptimal/under- 
controlled (Type 4) performance experiences. Data from the verbal reports suggest that braking control and "acceleration after the curve” are important for skilled performance in driving. Thus, in line with the expert performance approach (Williams \& Ericsson, 2005), scholars should consider analyzing the kinematic and psychophysiological mechanism of braking modulation and acceleration dynamics among skilled racecar drivers. Further, results support the notion that different perceived and psychophysiological states underlie the different MAP model categories. Specifically, we found differences among all MAP model categories for the drivers’ perceived emotional states and psychophysiological responses. However, it is important to note that the majority of the differences observed in the presented study were in the performance factor as related to optimal (Type 1 and/or Type 2) versus suboptimal performance experiences (Type 3 and/or Type 4). Overall, there is a general agreement that best and worst performance experiences are easier to distinguish (as opposed to differentiating near-optimal performance from optimal performance) because they are marked by distinct psychophysiological states expressed through different psychophysiological markers (e.g., muscle tension, heart rate; see Hanin, 2007; Robazza, 2006).

\section{Hypothesis 1: MAP's Model Performance Types}

Differences in all variables were observed when comparing suboptimal/over-controlled (Type 3) and suboptimal/under-controlled (Type 4) performance experiences. Although a trend could not be established (as drivers’ perceived and psychophysiological responses varied greatly), it was evident that high (Type 3) and low (Type 4) levels of attentional control resulted in different levels of accuracy on core components of action, hedonic tone, and psychophysiological responses. From an applied sport standpoint, profiling the dynamics of suboptimal performance experiences is crucial to increase the frequency of best performances. 
For instance, action-centered strategies (e.g., relaxation techniques) or attentional focused oriented strategies (e.g., attentional span and focus training) may be used to alter posture behavior according to one’s Type 1 performance profile (see Bertollo et al., 2013). For example, high levels of controlled focused attention could be beneficial to Driver 7 in maintaining a higher frequency of optimal performance experiences. Conversely, less attentional control as indicated by a more relaxed posture tone (i.e., leaning backwards as suggested by increased negative values) could help Driver 6 in moving from suboptimal performance types (Type 3 and Type 4) toward optimal performance states (Type 1 and Type 2). Additional applied strategies that can help athletes cope with fatigue feelings during long duration sport events, such as racecar driving, include associative and dissociative imagery (Hutchinson \& Karageorghis, 2013; Razon, Mandler, Arsal, Tokac, \& Tenenbaum, 2014). Overall findings suggest that increasing awareness on the accuracy of core components of action is beneficial to performance as this factor was significant for all drivers. Understanding the chain of events associated with optimal performance is paramount for the development of expert performance in sports (Ericsson, 2006). Differences between Type 1 and Type 2 performance were observed for accuracy on core components of action and heart rate only. Again, differences among various degrees of optimal performance states are usually subtle (Robazza, 2006). Furthermore, Type 1 performance states (optimal/automatic, flow-feeling like experiences) are rare occurrences and difficult to induce in totality, particularly in a laboratory setting. Perhaps key to help athletes moving toward Type 1 performance experiences are mindfulness approaches aimed at focusing on the moment ("here and now”) and at reducing judgmental thinking (Bortoli et al., 2012; Masters \& Maxwell, 2008). In effect, optimal-automatic performance experiences occur without overt conscious control through efficient parallel processing in the motor cortex (Comani et al., 2014a; Del Percio et al., 
2009). Finally, given that most athletes showed unique heart rate and respiratory rate patterns

2 linked to Type 1 performance, heart rate variability training could be beneficial in increasing the

3 likelihood of peak performance experiences. Indeed, this biofeedback technique has been used to

4 alter heart and respiratory functions in order to optimize performance in various domains of

5 human performance, including sports, physical rehabilitation, and military (Giggins et al., 2013). Hypothesis 2: Drivers’ Idiosyncratic Psychophysiological Responses

In agreement with extant idiosyncratic research in sport psychology (Edmonds et al., 2008; Filho et al., 2008), we observed large individual differences among the drivers.

9 Specifically, the differences were in the intensity, variability, and magnitude of the drivers' 10 subjective and psychophysiological recordings. These results are in accordance with the 11 overarching principle of individualization in athletic training. In this regard, some

12 psychophysiological markers (most noticeably respiratory rate and skin temperature) were

13 predictors of performance experiences for only a few drivers. In fact, Hanin's (2007) pentagram

14 conceptualization within the individual zones of optimal functioning model predicts that

15 different athletes are more or less sensitive to different forms of psychophysiological

16 intervention. Therefore, we reinforce the importance of idiosyncratic research in sport

17 psychology, particularly among skilled athletes. Adhering to normalized standards and nomothetic analysis (averaging data across participants) can be misleading in identifying the

19 unique core components of optimal performance for a given athlete (Edmonds et al., 2008; Filho et al., 2008; Kamata et al., 2002). In all, we echo the notion that multimodal assessment plans

21 and intervention protocols should be designed to allow athletes to choose among multi-action 22 plans depending on situational factors and the task at hand. 


\section{Limitations and Future Directions}

It is important to highlight that the present study has limitations. First, it is difficult to induce Type 1, flow-like performance in laboratory settings (Kimiecik \& Jackson, 2002). Peak performance experiences are rare, and hence pose a challenge to scholars and practitioners interested in its nomological network. Second, the diversity in age and competitive background of the sampled athletes may explain part of the variability found in their subjective and psychophysiological responses. Third, testing for interactions among the variables was beyond the scope of this study, which focused on identifying the unique psychophysiological channels linked to optimal and suboptimal performance experiences in racecar driving. Examining the moderating and mediating linkage among various physiological measures, such as heart rate, skin conductance, and electroencephalographic patterns, represents the next step in advancing research on bio-neurofeedback training protocols. Finally, although we used a professional race simulator, it is not possible to fully replicate an actual racecar competition. In fact, athletes and their staff are usually less inclined to participate in "real-world" data collection due to the inherently dangerous nature of motorsports (Fuller, 2005). When available, G-force simulators should be used to better replicate the physical properties of real-world races.

Notwithstanding these limitations, our study expands research in motorsports through a multimodal yet idiosyncratic approach. Most previous studies in motorsports have integrated only two psychophysiological measures and performance data. In the present study, we used four measures while simultaneously assessing drivers’ performance (Katsis et al., 2008). Additionally, we expanded research on the MAP model, which in the past has been primarily conducted in self-paced sports (Bertollo et al., 2013; Bortoli et al., 2012). Moreover, we were able to monitor highly skilled racecar drivers, whose career highs included top-3 placements in 
major European competitions. As alluded to previously, it is crucial to study skilled athletes in order to advance research on the mediating mechanisms (e.g., physiological markers, memory structures) of expert performance in sports (Ericsson, 2006). Also noteworthy, this study adds to the literature in motorsports. Motorsports are less studied in comparison to other sports due to their dangerous nature and because drivers are not perceived as athletes by those who believe that the car is the most important factor in racing (Potkanowicz \& Mendel, 2013). This study also adds to the extant literature on optimal performance experiences in sport psychology, especially in regards to the underpinning subjective and psychophysiological mechanisms differentiating optimal from suboptimal performance experiences.

Experimental trials are needed to advance knowledge on the MAP model's $2 \times 2$ (performance $\times$ attentional focus) categorization. Future studies should assess changes in the ability of maintaining a Type 1 performance state after an action-centered and/or attentionfocused training regime. Qualitatively contrasting athletes and coaches mental models on the core components of action in a given sport may help in the development of applied strategies aimed at enhancing performance in sports. Nomothetic research based on large samples may help to describe the psychophysiological mechanisms explaining the variability on drivers’ raw performance data and psychophysiological responses. For instance, nomothetic research may help to explain why heart rate patterns are higher for some drivers and lower for other drivers across performance types. Kinematic and high-definition video analysis may be used to objectively evaluate athletes' core components of action in both closed and open skill sports. Finally, as outlined elsewhere (Del Percio et al., 2009), scholars should continue to explore the neural-efficiency hypothesis (common in optimal-automatic experiences) through the use of electroencephalographic and near-infrared spectroscopy methodologies. 


\section{Conclusion}

In summary, our findings are consistent with previous research on the MAP model in

3 which athletes' psychophysiological states were found to differ as a function of distinct

4 performance levels (i.e., optimal- suboptimal) and attentional demands (i.e., automatic-

5 controlled). Results are also aligned with applied research in sport psychology in regards to the

6 importance of developing idiosyncratic and multimodal plans for performance optimization in

7 sports. Specifically, developing action-centered strategies (e.g., brake modulation control) and

8 attention-focused strategies (e.g., attentional focus directed at the "racing line”) may help

9 athletes move toward less controlled, more pleasant, and overall better performance states in

10 racecar driving. Further, bio-neurofeedback training regimes may help athletes regulate their

11 psychophysiological states, thus increasing their probability of peak performance. 


\section{References}

Bertollo, M., Bortoli, L., Gramaccioni, G., Hanin, Y., Comani, S., \& Robazza, C. (2013). Behavioural and psychophysiological correlates of athletic performance: A test of the multi-action plan model. Applied Psychophysiology and Biofeedback, 38, 91-99. doi: $10.1007 / \mathrm{s} 10484-013-9211-\mathrm{z}$

Bertollo, M., Robazza, C., Falasca, W. N., Stocchi, M., Babiloni, C., Del Percio, C., ...Comani, S. (2012). Temporal pattern of pre-shooting psycho-physiological states in elite athletes: A probabilistic approach. Psychology of Sport and Exercise, 13, 91-98. doi: 10.1016/j.psychsport.2011.09.005

Borg, G. (2001). Borg's range model and scales. International Journal of Sport Psychology, 32, 110-126.

Bortoli, L., Bertollo, M., Hanin, Y., \& Robazza, C. (2012). Striving for excellence: A multiaction plan intervention model for shooters. Psychology of Sport and Exercise, 13, 693701. doi: 10.1016/j.psychsport.2012.04.006

Brearley, M. B., \& Finn, J. P. (2007). Responses of motor-sport athletes to V8 supercar racing in hot conditions. International Journal of Sports Physiology and Performance, 2, 182-191.

Comani, S., Bortoli, L. Di Fronso, S., Filho, E., De Marchis, C., M., Schmid, M., Bortoli, L., ...Bertollo, M. (2014a). ERD/ERS patterns of shooting performance within the multiaction plan model. International Federation for Medical and Biological Engineering, 41, 131-144.

Comani, S., Di Fronso, S., Filho, E., De Marchis, C., Schmid, M., Bortoli, L., ...Bertollo, M. (2014b). Attentional focus and functional connectivity in cycling: An EEG case study. International Federation for Medical and Biological Engineering, 41, 137-140. 
Del Percio, C., Babiloni, C., Bertollo, M., Marzano, N., Iacoboni, M., Infarinato, F., ... Eusebi, F. (2009). Visuo-attentional and sensorimotor alpha rhythms are related to visuo-motor performance in athletes. Human Brain Mapping, 30, 3527-3540. doi: 10.1002/hbm.20776

Edmonds, W. A., Tenenbaum, G., Mann, D. Y., Johnson, M., \& Kamata, A. (2008). The effect of biofeedback training on affective regulation and simulated car-racing performance: A multiple case study analysis. Journal of Sports Sciences, 26, 761-773. doi: $10.1080 / 02640410701813068$

Ericsson, K. A. (2006). Protocol analysis and expert thought: Concurrent verbalizations of thinking during experts’ performance on representative tasks. In K. A. Ericsson, N. Charness, P. J. Feltovich, \& R. R. Hoffman (Eds.), Cambridge handbook of expertise and expert performance (pp. 223-242). New York, NY: Cambridge University Press.

Filho, E., Moraes, L. C., \& Tenenbaum, G. (2008). Affective and physiological states during archery competitions: Adopting and enhancing the probabilistic methodology of individual affect-related performance zones (IAPZs). Journal of Applied Sport Psychology, 20, 441-456. doi: 10.1080/10413200802245221

Fuller, R. (2005). Towards a general theory of driver behaviour. Accident Analysis and Prevention, 37, 461-472. doi: 10.1016/j.aap.2004.11.003

Gardner, F. L, \& Moore, Z. E. (2004). A Mindfulness-Acceptance-Commitment-based approach to athletic performance enhancement: Theoretical considerations. Behavior Therapy, 35, 707-723.

Giggins, O. M., Persson, U. M., \& Caulfield, B. (2013). Biofeedback in rehabilitation. Journal of Neuroengineering and Rehabilitation, 10, 1-11. doi: 10.1186/1743-0003-10-60 
Hanin, Y. L. (2007). Emotions in sport: Current issues and perspectives. In G. Tenenbaum, \& R. C. Eklund (Eds.), Handbook of sport psychology (3rd ed., pp. 31-58). Hoboken, NJ: Wiley \& Sons.

Hanin, Y., \& Hanina, M., with the Commentators (2009). Optimization of performance in toplevel athletes: An action-focused coping approach. International Journal of Sports Science and Coaching, 4, 47-91.

Holland, C., Geraghty, J., \& Shah, K. (2010). Differential moderating effect of locus of control on effect of driving experience in young male and female drivers. Personality and Individual Differences, 48, 821-826. doi: 10.1016/j.paid.2010.02.003

Hutchinson, J. C., \& Karageorghis, C. I. (2013). Moderating influence of dominant attentional style and exercise intensity on responses to asynchronous music. Journal of Sport and Exercise Psychology, 35, 625-643.

Johnson, M. B., Edmonds, W. A., Moraes, L. C., Filho, E. S., \& Tenenbaum, G. (2007). Linking affect and performance of an international level archer incorporating an idiosyncratic probabilistic method. Psychology of Sport and Exercise, 8, 317-335. doi: 10.1016/j.psychsport.2006.05.004

Kamata, A., Tenenbaum, G., \& Hanin, Y. L. (2002). Individual Zone of Optimal Functioning (IZOF): A probabilistic estimation. Journal of Sport and Exercise Psychology, 24, 189208.

Katsis, C. D., Katertsidis, N., Ganiatsas, G., \& Fotiadis, D. I. (2008). Toward emotion recognition in car-racing drivers: A biosignal processing approach. IEEE Transactions on Systems, Man, and Cybernetics, Part A, 502-512. 
Kimiecik, J. C., \& Jackson, S. A. (2002). Optimal experience in sport: A flow perspective. In T. S. Horn (Ed.), Advances in sport psychology (2nd ed., pp. 501-527). Champaign, IL: Human Kinetics.

Masters, R., \& Maxwell, J. (2008). The theory of reinvestment. International Review of Sport and Exercise Psychology, 1, 160-183. doi: 10.1080/17509840802287218.

Mclaren Mercedes (2013). The Perfect Lap. United Kingdom: Mclaren Studio.

Mullen, R., Jones, E. S., Faull, A., \& Kingston, K. (2012). Attentional focus and performance anxiety: effects on simulated race-driving performance and heart rate variability. Frontiers in Psychology, 3, Article 426, 1-10. doi: 10.3389/fpsyg.2012.00426

Patton, M. Q. (2002). Qualitative research and evaluation methods (3rd ed.). Thousand Oaks, CA: Sage.

Potkanowicz, E., \& Mendel, R. (2013). The case for driver science in motorsport: A review and recommendations. Sports Medicine, 43, 565-574. doi: 10.1007/s40279-013-0040-2

Razon, S., Mandler, K., Arsal, G., Tokac, U., \& Tenenbaum, G. (2014). Effects of imagery on effort perception and endurance. Journal of Imagery Research in Sport and Physical Activity. doi: 10.1515/jirspa-2013-0011

Robazza, C. (2006). Emotion in sport: An IZOF perspective. In S. Hanton \& S. D. Mellalieu (Eds.), Literature reviews in sport psychology (pp. 127-158). NY: Nova Science.

Robazza, C., Bertollo, M., Hanin, Y., Filho, E., \& Bortoli, L. (2014). What about (dis)pleasure and (dis)comfort while performing in sport? Submitted.

Russell, J. A., Weiss, A., \& Mendelsohn, G. A. (1989). Affect grid: A single-item scale of pleasure and arousal. Journal of Personality and Social Psychology, 57, 493-502. doi: 10.1037/0022-3514.57.3.493. 
Tenenbaum, G., Basevitch, I., Gershgoren, L., \& Filho, E. (2013). Emotions-decision-making in sport: Theoretical conceptualization and experimental evidence. International Journal of Sport and Exercise Psychology, 11, 151-168. doi: 10.1080/1612197X.2013.773687

Vickers, J. N. (2006). Gaze of Olympic speed skaters while skating at full speed on regulation oval: Perception-action coupling in a dynamic performance environment. Cognitive Processing, 7, 102-105. doi: 10.1007/s10339-006-0087-1

Walker, S. M., Dawson, B., \& Ackland, T. R. (2001). Performance enhancement in rally car drivers via heat acclimation and race simulation. Comparative Biochemistry and Physiology a-Molecular and Integrative Physiology, 128, 701-707. doi: 10.1016/s10956433(01)00276-8

Williams, A. M., \& Ericsson, K. A. (2005). Perceptual-cognitive expertise in sport: Some considerations when applying the expert performance approach. Human Movement Science, 24, 283-307. doi: 10.1016/j.humov.2005.06.002

Yamakoshi, T., Matsumura, K., Yamakoshi, Y., Hirose, H., \& Rolfe, P. (2010). Physiological measurements and analyses in motor sports: A preliminary study in racing kart athletes. European Journal of Sport Science, 10, 397-406. doi: 10.1080/17461391003699112 
Table 1

Descriptive and Inferential Analysis of Drivers' Accuracy on Core Components of Action (CCA) and Hedonic Tone

\begin{tabular}{|c|c|c|c|c|c|c|c|}
\hline Driver & Type 1 (T1) & Type 2 (T2) & Type 3 (T3) & Type 4 (T4) & $F(3,117)$ & $d$ & Post-Hoc \\
\hline 1 & $n=35$ & $n=25$ & $n=38$ & $n=22$ & & & \\
\hline Accuracy CCA & $6.23(1.39)$ & $5.28(2.30)$ & $5.55(1.45)$ & $6.52(1.12)$ & $3.50^{*}$ & 0.31 & $\mathrm{~T} 2<\mathrm{T} 4$ \\
\hline Hedonic Tone & $5.66(2.66)$ & 4.32 (3.87) & $5.08(2.63)$ & 6.35 (1.92) & 2.80 & & \\
\hline 2 & $n=15$ & $n=45$ & $n=21$ & $n=39$ & & & \\
\hline Accuracy CCA & $6.67(1.40)$ & $8.22(0.97)$ & $6.48(2.27)$ & $4.33(2.07)$ & $36.56^{* *}$ & 0.14 & $\mathrm{~T} 1>\mathrm{T} 3 ; \mathrm{T} 1>\mathrm{T} 4 ; \mathrm{T} 2>\mathrm{T} 3$ \\
\hline $\begin{array}{c}\text { Hedonic Tone } \\
3\end{array}$ & $\begin{array}{c}1.27(5.40) \\
n=38\end{array}$ & $\begin{array}{c}5.84(2.90) \\
n=26\end{array}$ & $\begin{array}{c}2.57(5.98) \\
n=32\end{array}$ & $\begin{array}{c}-3.41(6.51) \\
n=24\end{array}$ & $22.54^{* *}$ & 1.39 & $\mathrm{~T} 1<\mathrm{T} 2 ; \mathrm{T} 2>\mathrm{T} 4$ \\
\hline Accuracy CCA & $6.79(1.17)$ & $6.69(2.11)$ & $4.56(2.67)$ & $4.67(3.36)$ & $7.58^{* *}$ & 0.71 & $\mathrm{~T} 1>\mathrm{T} 3 ; \mathrm{T} 1>\mathrm{T} 4 ; \mathrm{T} 2>\mathrm{T} 3 ; \mathrm{T} 2>\mathrm{T} 4$ \\
\hline $\begin{array}{l}\text { Hedonic Tone } \\
4\end{array}$ & $\begin{array}{c}6.13(3.54) \\
n=38\end{array}$ & $\begin{array}{c}4.38(5.31) \\
n=22\end{array}$ & $\begin{array}{c}0.78(6.50) \\
n=26\end{array}$ & $\begin{array}{c}3.38(5.03) \\
n=34\end{array}$ & $6.56^{* *}$ & 0.78 & $\mathrm{~T} 1>\mathrm{T} 3$ \\
\hline Accuracy CCA & $7.42(0.83)$ & 7.09 (1.66) & $6.38(1.30)$ & $6.97(1.06)$ & $4.00^{* *}$ & 0.51 & $\mathrm{~T} 1>\mathrm{T} 3$ \\
\hline $\begin{array}{l}\text { Hedonic Tone } \\
5\end{array}$ & $\begin{array}{c}5.00(2.05) \\
n=51\end{array}$ & $\begin{array}{c}5.09(1.57) \\
n=09\end{array}$ & $\begin{array}{c}2.92(2.62) \\
n=09\end{array}$ & $\begin{array}{c}2.62(2.84) \\
n=51\end{array}$ & $9.46^{* *}$ & 0.69 & T1>T3; T1>T4; T2> T3; T2>T4 \\
\hline Accuracy CCA & $2.41(1.79)$ & $3.78(1.30)$ & $2.67(2.35)$ & $0.63(1.55)$ & $15.09^{* *}$ & 0.65 & T1>T4; T2>T4; T3>T4 \\
\hline $\begin{array}{c}\text { Hedonic Tone } \\
6\end{array}$ & $\begin{array}{c}-0.22(1.36) \\
n=35\end{array}$ & $\begin{array}{c}0.78(0.83) \\
n=25\end{array}$ & $\begin{array}{c}-1.56(3.71) \\
n=38\end{array}$ & $\begin{array}{c}-0.71(1.88) \\
n=22\end{array}$ & $3.10^{* *}$ & 0.93 & $\mathrm{~T} 2>\mathrm{T} 3$ \\
\hline Accuracy CCA & $6.23(1.40)$ & $5.28(2.30)$ & $5.55(1.45)$ & $6.64(1.00)$ & $3.97^{* *}$ & 0.30 & $\mathrm{~T} 2<\mathrm{T} 4$ \\
\hline $\begin{array}{c}\text { Hedonic Tone } \\
7\end{array}$ & $\begin{array}{c}5.66(2.66) \\
n=31\end{array}$ & $\begin{array}{c}4.32(3.87) \\
n=29\end{array}$ & $\begin{array}{c}5.08(2.63) \\
n=38\end{array}$ & $\begin{array}{c}6.45(1.90) \\
n=22\end{array}$ & 2.47 & & \\
\hline Accuracy CCA & $7.94(1.61)$ & $7.66(0.86)$ & $6.32(2.00)$ & $7.41(1.62)$ & $6.79^{* *}$ & 0.58 & $\mathrm{~T} 1>\mathrm{T} 3 ; \mathrm{T} 2>\mathrm{T} 3$ \\
\hline $\begin{array}{c}\text { Hedonic Tone } \\
8\end{array}$ & $\begin{array}{c}7.87(1.76) \\
n=16\end{array}$ & $\begin{array}{c}7.31(1.14) \\
n=42\end{array}$ & $\begin{array}{c}3.95(4.60) \\
n=30\end{array}$ & $\begin{array}{c}6.27(3.34) \\
n=32\end{array}$ & $10.74^{* *}$ & 0.74 & T1>T3; T2>T3; T3<T4 \\
\hline Accuracy CCA & $4.06(3.06)$ & $6.20(1.94)$ & $6.43(1.77)$ & $0.18(5.77)$ & $22.76^{* *}$ & 0.09 & $\mathrm{~T} 1>\mathrm{T} 4 ; \mathrm{T} 2>\mathrm{T} 4 ; \mathrm{T} 3>\mathrm{T} 4$ \\
\hline $\begin{array}{c}\text { Hedonic Tone } \\
9\end{array}$ & $\begin{array}{c}-0.31(0.79) \\
n=35\end{array}$ & $\begin{array}{c}-0.07(0.89) \\
n=25\end{array}$ & $\begin{array}{c}0.07(0.87) \\
n=47\end{array}$ & $\begin{array}{c}0.24(0.97) \\
n=13\end{array}$ & 1.58 & & \\
\hline Accuracy CCA & $9.17(1.48)$ & $6.58(2.59)$ & $5.77(2.91)$ & $6.15(3.24)$ & $12.90^{*}$ & 1.22 & $\mathrm{~T} 1>\mathrm{T} 2 ; \mathrm{T} 1>\mathrm{T} 3 ; \mathrm{T} 1>\mathrm{T} 4$ \\
\hline Hedonic Tone & $4.86(2.96)$ & $2.08(3.43)$ & $1.04(3.49)$ & $1.00(4.08)$ & $9.40^{*}$ & 1.03 & $\mathrm{~T} 1>\mathrm{T} 2 ; \mathrm{T} 1>\mathrm{T} 3 ; \mathrm{T} 1>\mathrm{T} 4$ \\
\hline
\end{tabular}

$* p<.05 . * * p<.01$. 
Table 2

Descriptive and Inferential Analysis of Drivers Psychophysiological Variables

\begin{tabular}{|c|c|c|c|c|c|c|c|}
\hline Driver & Type 1 (T1) & Type 2 (T2) & Type 3 (T3) & Type 4 (T4) & $F(3,117)$ & $d$ & Post-Hoc \\
\hline 1 & $n=35$ & $n=25$ & $n=38$ & $n=22$ & & & \\
\hline HR & $99.77(3.06)$ & $101.00(2.20)$ & $103.97(2.27)$ & 96.95 (17.96) & $3.85^{*}$ & 0.16 & $\mathrm{~T} 3>\mathrm{T} 4$ \\
\hline RR & $21.47(2.56)$ & $22.90(2.49)$ & $24.08(2.35)$ & $22.76(4.30)$ & $4.90^{* *}$ & 0.66 & $\mathrm{~T} 1<\mathrm{T} 3$ \\
\hline $\mathrm{ST}$ & $36.67(0.89)$ & $36.74(0.09)$ & $36.00(0.67)$ & $36.54(0.26)$ & $24.65^{* *}$ & 0.78 & T1>T3; T2> T3; T3<T4 \\
\hline PT & $-17.03(0.86)$ & $-17.39(0.97)$ & $-19.23(1.36)$ & $-16.72(3.88)$ & $11.23^{* *}$ & 0.52 & T1>T3; T2>T3; T3<T4 \\
\hline 2 & $n=15$ & $n=45$ & $n=21$ & $n=39$ & & & \\
\hline HR & $70.83(4.14)$ & $72.53(3.61)$ & $70.75(2.36)$ & $68.34(5.63)$ & $4.08^{* *}$ & 0.04 & $\mathrm{~T} 2>\mathrm{T} 4$ \\
\hline $\mathrm{RR}$ & $14.78(6.40)$ & $16.05(5.49)$ & 15.54 (4.99) & $15.74(4.43)$ & $2.70^{*}$ & 0.70 & $\mathrm{~T} 1<\mathrm{T} 2$ \\
\hline $\mathrm{ST}$ & $34.94(0.79)$ & $34.93(0.89)$ & $34.87(0.15)$ & $34.87(0.12)$ & 2.86 & & \\
\hline PT & $-15.05(1.63)$ & $-15.22(1.43)$ & $-16.21(2.13)$ & $-16.11(1.71)$ & $3.34^{*}$ & 0.42 & $\mathrm{~T} 2>\mathrm{T} 4$ \\
\hline 3 & $n=38$ & $n=26$ & $n=32$ & $n=24$ & & & \\
\hline HR & $87.45(7.98)$ & $82.54(5.15)$ & $84.72(7.41)$ & $84.93(5.35)$ & $2.77^{*}$ & 0.52 & $\mathrm{~T} 1>\mathrm{T} 2$ \\
\hline $\mathrm{RR}^{\dagger}$ & - & - & - & - & - & - & - \\
\hline ST & $36.02(0.31)$ & $35.74(0.39)$ & $35.72(0.49)$ & $35.81(0.40)$ & $4.08^{* *}$ & 0.67 & $\mathrm{~T} 1>\mathrm{T} 3$ \\
\hline PT & $-9.22(1.50)$ & $-10.10(1.48)$ & $-10.32(2.24)$ & $-9.59(1.63)$ & $2.70^{*}$ & 0.47 & $\mathrm{~T} 1>\mathrm{T} 3$ \\
\hline 4 & $n=38$ & $n=22$ & $n=26$ & $n=34$ & & & \\
\hline HR & $88.47(5.15)$ & $90.61(5.28)$ & 82.39 (3.69) & $83.95(5.72)$ & $15.20^{* *}$ & 0.64 & $\mathrm{~T} 1>\mathrm{T} 3 ; \mathrm{T} 1>\mathrm{T} 4 ; \mathrm{T} 2>\mathrm{T} 3 ; \mathrm{T} 2>\mathrm{T} 4$ \\
\hline $\mathrm{RR}$ & $15.26(3.17)$ & $13.93(2.21)$ & $15.35(2.46)$ & $15.00(2.41)$ & 1.45 & & \\
\hline $\mathrm{ST}$ & $36.68(0.23)$ & $36.56(0.56)$ & $35.42(1.15)$ & $36.26(0.82)$ & $16.54^{* *}$ & 0.82 & $\mathrm{~T} 1>\mathrm{T} 3 ; \mathrm{T} 2>\mathrm{T} 3 ; \mathrm{T} 3<\mathrm{T} 4$ \\
\hline PT & $-21.29(0.81)$ & $-21.53(0.80)$ & $-20.82(0.48)$ & $-20.88(0.71)$ & $5.88^{* *}$ & 0.35 & $\mathrm{~T} 2<\mathrm{T} 3 ; \mathrm{T} 2<\mathrm{T} 4$ \\
\hline 5 & $n=51$ & $n=09$ & $n=51$ & $n=09$ & & & \\
\hline HR & $76.61(5.22)$ & $76.22(3.22)$ & $79.40(3.65)$ & $74.85(4.42)$ & $2.95^{*}$ & 0.21 & $\mathrm{~T} 3>\mathrm{T} 4$ \\
\hline RR & $21.74(1.80)$ & $22.60(1.70)$ & 22.22 (2.09) & $21.33(2.30)$ & 1.37 & & \\
\hline $\mathrm{ST}$ & $36.44(0.20)$ & $36.34(0.20)$ & $36.27(0.21)$ & $36.44(0.19)$ & 2.53 & & \\
\hline $\mathrm{PT}$ & $-26.50(0.20)$ & $-27.03(0.67)$ & $-27.08(0.69)$ & $-26.59(0.83)$ & 2.08 & & \\
\hline
\end{tabular}


Table 2 Continued

Descriptive and Inferential Analysis of Drivers Psychophysiological Variables

\begin{tabular}{|c|c|c|c|c|c|c|c|}
\hline Driver & Type 1 (T1) & Type 2 (T2) & Type 3 (Т3) & Type 4 (T4) & $F(3,117)$ & $d$ & Post-Hoc \\
\hline 6 & $n=35$ & $n=25$ & $n=38$ & $n=22$ & & & \\
\hline HR & $105.60(1.77)$ & $101.87(1.49)$ & $100.90(2.39)$ & $97, .9(1.88)$ & $64.23^{* *}$ & 2.40 & T1>T2; T1>T3; T1>T4; T2>T4; T3>T4 \\
\hline $\mathrm{RR}$ & $15.43(2.50)$ & $23.35(2.01)$ & $23.11(2.20)$ & 19.71(2.90) & $14.53^{* *}$ & 1.22 & $\mathrm{~T} 1<\mathrm{T} 4 ; \mathrm{T} 2<\mathrm{T} 4 ; \mathrm{T} 3<\mathrm{T} 4$ \\
\hline ST & $34.55(0.62)$ & $36.79(0.06)$ & $36.69(0.04)$ & $36.58(0.02)$ & $50.41^{* *}$ & 2.46 & $\mathrm{~T} 1<\mathrm{T} 2 ; \mathrm{T} 1<\mathrm{T} 3 ; \mathrm{T} 1<\mathrm{T} 4$ \\
\hline PT & $-21.96(0.84)$ & $-18.12(0.63)$ & $-16.87(0.59)$ & $-16.48(0.77)$ & $131.81^{* *}$ & 3.87 & $\mathrm{~T} 1<\mathrm{T} 2 ; \mathrm{T} 1<\mathrm{T} 3 ; \mathrm{T} 1<\mathrm{T} 4 ; \mathrm{T} 2<\mathrm{T} 3 ; \mathrm{T} 2<\mathrm{T} 4$ \\
\hline 7 & $n=31$ & $n=29$ & $n=38$ & $n=22$ & & & \\
\hline HR & 85.08 (7.33) & $87.20(6.81)$ & $84.35(6.35)$ & $82.27(4.21)$ & 2.57 & & \\
\hline $\mathrm{RR}^{\dagger}$ & - & - & - & - & & & \\
\hline ST & $35.99(0.27)$ & $36.02(0.22)$ & $35.70(0.50)$ & $35.55(0.41)$ & $10.31^{* *}$ & 1.12 & $\mathrm{~T} 2>\mathrm{T} 4$ \\
\hline PT & $-9.17(1.28)$ & $-9.06(1.22)$ & $-10.45(2.37)$ & $-10.60(1.51)$ & $6.49^{* *}$ & 0.50 & T1>T3; T1>T4; T2>T3; T2>T4 \\
\hline 8 & $n=16$ & $n=42$ & $n=30$ & $n=32$ & & & \\
\hline HR & $77.58(2.55)$ & $78.77(2.65)$ & 77.55 (3.21) & $78.77(2.51)$ & 1.83 & & \\
\hline $\mathrm{RR}$ & $19.74(4.91)$ & $18.98(3.95)$ & $18.52(3.34)$ & $20.26(4.15)$ & 1.15 & & \\
\hline ST & $36.48(0.15)$ & $36.56(0.16)$ & $36.53(0.17)$ & $36.58(0.15)$ & 1.78 & & \\
\hline PT & $-23.16(0.48)$ & $-23.24(0.42)$ & $-23.03(0.53)$ & $-23.21(0.40)$ & 1.43 & & \\
\hline 9 & $n=35$ & $n=25$ & $n=47$ & $n=13$ & & & \\
\hline HR & $101.91(3.23)$ & $102.72(2.79)$ & 101.83 (2.84) & $101.78(3.60)$ & 0.56 & & \\
\hline $\mathrm{RR}$ & $18.73(3.29)$ & $19.66(4.67)$ & $19.70(3.36)$ & $18.57(3.62)$ & 0.72 & & \\
\hline ST & $36.48(0.14)$ & $36.31(0.47)$ & 35.98 (0.69) & $36.30(0.28)$ & $7.21^{* *}$ & 0.71 & T1>T3; T2>T3 \\
\hline PT & $-19.81(1.42)$ & $-20.06(1.17)$ & $-20.39(2.17)$ & $-23.22(1.29)$ & 0.81 & & \\
\hline
\end{tabular}

Note. Heart rate (HR), respiratory rate (RR), skin temperature (ST), and posture (PT). ${ }^{\dagger}$ Missing Data. 


\section{High}

\section{Type 2 performance}

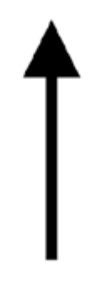

\section{Type 1 performance} Deliberate control

(1) Outomated control

High $\longleftarrow$ Attention Excessive control (

$\frac{E}{\mathrm{C}}$ control

Lack of control

Type 3 performance

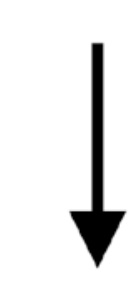

Type 4 performance

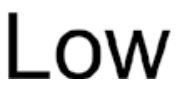

Figure 1. Performance and attention control interaction according to the multi-action plan (MAP) model. 

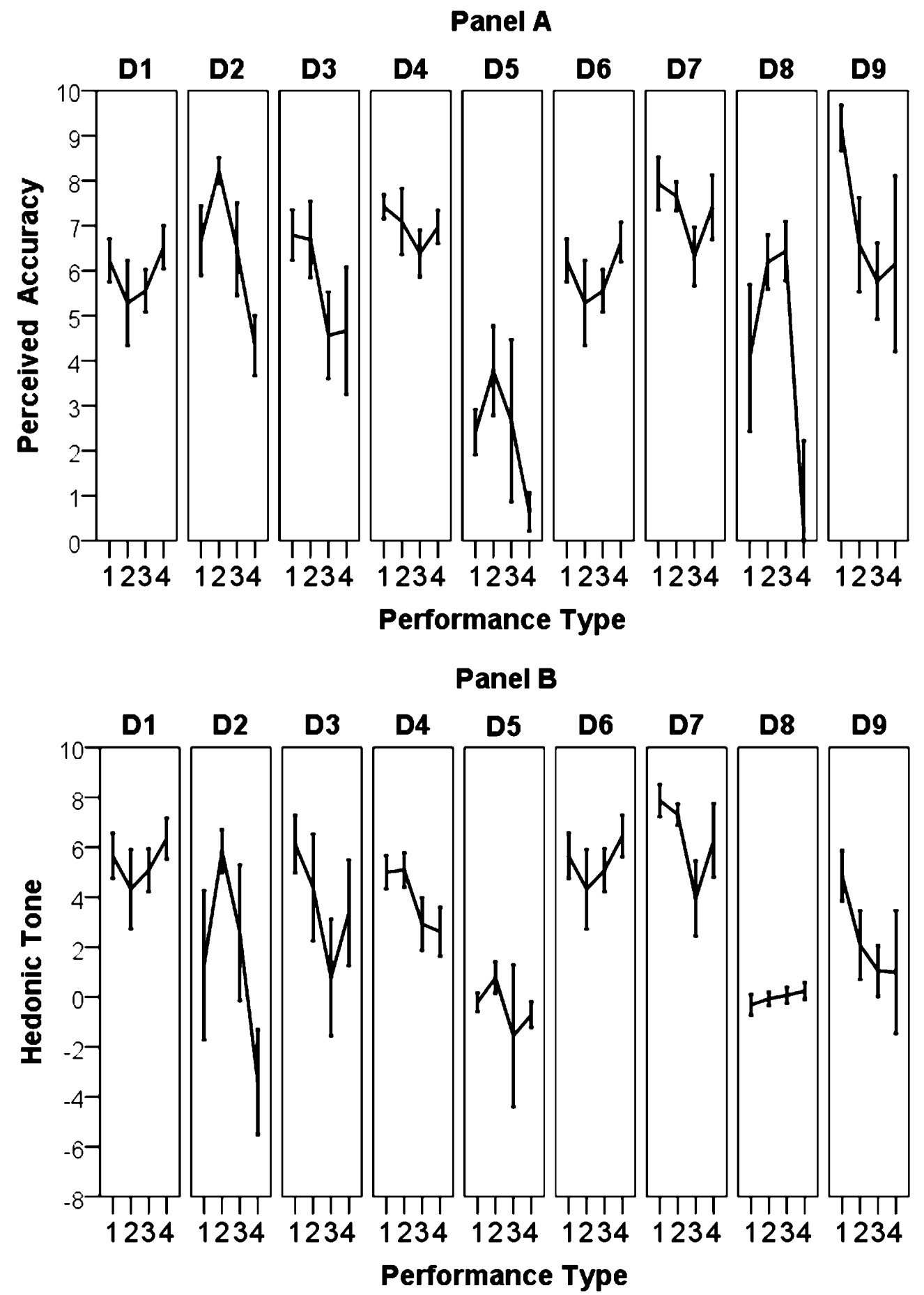

Figure 2. Drivers' perceived accuracy on core components of action (upper panel) and hedonic tone (lower panel) based on the MAP model framework.

Note. “D” stands for “Driver”. X-Axis: Performance Type-1, Type-2, Type-3, and Type-4. 

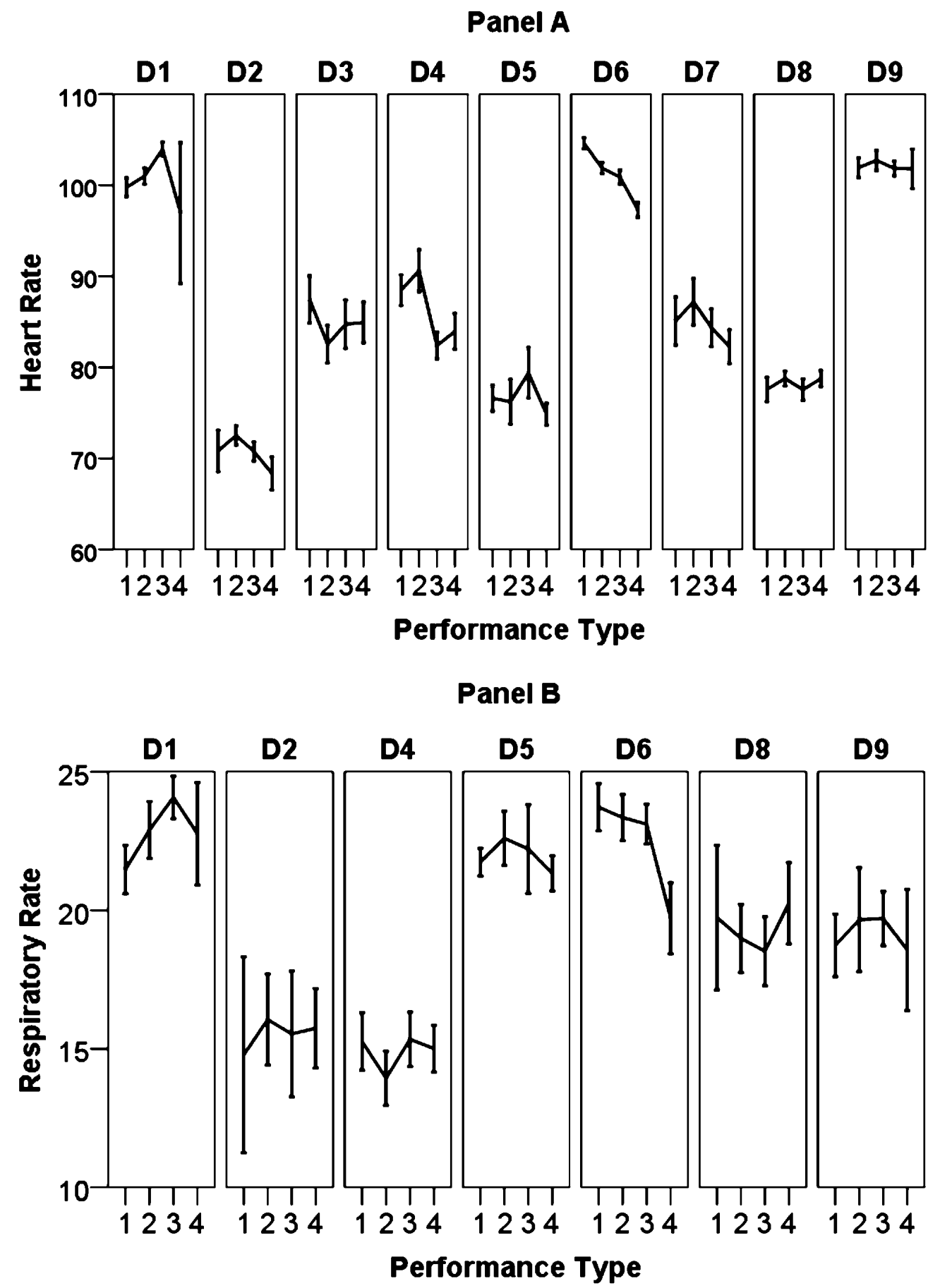

Figure 3. Drivers' heart rate (HR; bpm) and respiratory rate (RR; breaths per minute) based on the MAP model framework.

Note. "D” stands for “Driver”. X-Axis: Performance Type-1, Type-2, Type-3, and Type-4. 

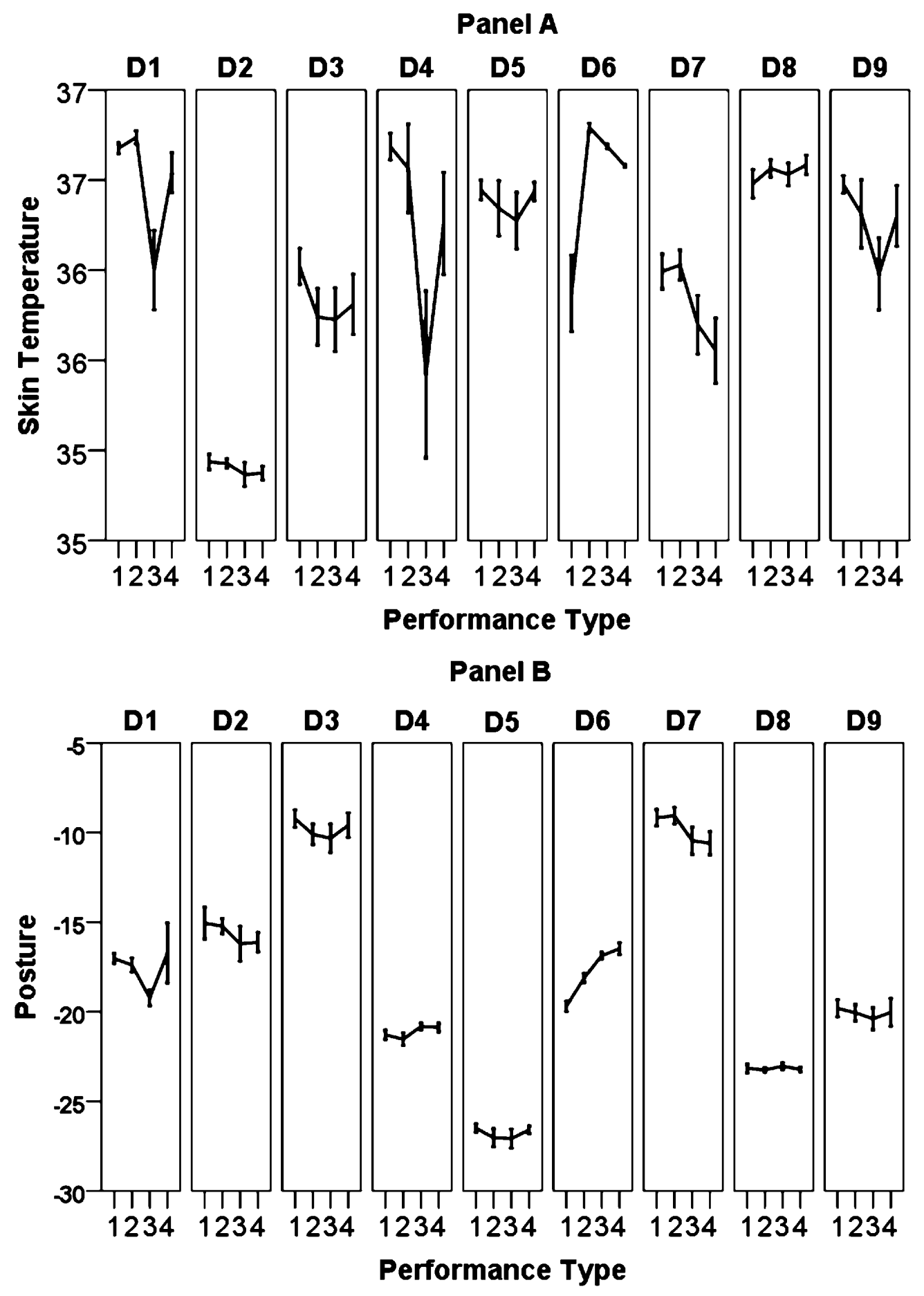

Figure 4. Drivers' skin temperature (ST; Celsius) and posture (PT) based on the MAP model framework.

Note. "D” stands for “Driver”. X-Axis: Performance Type-1, Type-2, Type-3, and Type-4. 